\title{
CANDIDATE MEDICINAL PLANT SPECIES OF DJIBOUTIAN PHARMACOPEIA FOR TESTING PHARMACOLOGICAL ACTIVITIES ON COMMON MICROBIAL DISEASES
}

\author{
FATOUMA MOHAMED ABDOUL-LATIF ${ }^{*}$, DJALTOU ABOUBAKER OSMAN ${ }^{1}$, ABDIRAHMAN ELMI FOURREH ${ }^{1}$, \\ ALSHAIMAA HASSAN-ABDALLAH ${ }^{1}$, ALI MERIT0 ${ }^{1}$, SOUAD HASSAN ${ }^{1}$, ZEMEDE ASFAW ${ }^{2}$, ENSERMU KELBESSA ${ }^{2}$
}

${ }^{1}$ Medicinal Research Institute, Centre d'Etudes et de Recherche de Djibouti, Route de L'aéroport, BP: 486 Djibouti, Djibouti, ${ }^{2}$ Department of Plant Biology and Biodiversity Management, College of Natural Sciences, Addis Ababa University, P. 0. Box 3434, Addis Ababa, Ethiopia Email: fatouma_abdoulatif@yahoo.fr

Received: 19 Mar 2015 Revised and Accepted: 12 Aug 2016

\begin{abstract}
Objective: The aim of the study was to conduct an ethnobotanical study focused on the medicinal plants used in Randa (Djibouti) for testing pharmacological activities on common microbial diseases.

Methods: Plant Ratio (PR), Index of Performance (IP) and the high Informant Consensus Factors (ICF) were calculated to select candidate medicinal species with a pharmacological potential.

Results: From this previous work, it was found that the high Informant Consensus Factors, ICF (0.82) for the category of microbial diseases turned out to merit further perusal. The findings particularly gave an imminent insight that stimulated additional investigations and analyses. Different factors, including Plant Ratio (PR) and Index of Performance (IP) were employed for comparison leading to the selection of 18 candidates species, for subsequent pharmacological screening, and testing for antimicrobial activities. Comparison of the information with that accessed from the literature implicitly hinted that the Djiboutian traditional medical system shares much in common with other traditional medical systems. At least six out of the 18 species have not been pharmacologically tested before.
\end{abstract}

Conclusion: The comparison of differents factors (IP2, PR and FL') of plants screening have showed that 18 plants species of Randa region, have good healing potential for infectious diseases. This may inspire continued research to build a comprehensive ethnobotanical and ethnopharmacological profiles of the species anticipated to be of the most promising potential for Djibouti and beyond.

Keywords: Traditional medicinal plants, Djibouti, Randa, Statistical analysis

(C) 2016 The Authors. Published by Innovare Academic Sciences Pvt Ltd. This is an open access article under the CC BY license (http://creativecommons. org/licenses/by/4. 0/) DOI: http://dx.doi.org/10.22159/ijpps.2016v8i10.5788

\section{INTRODUCTION}

Many clinically useful prescription drugs have originated from traditional medicinal plants. Since there are many traditional medicinal plants widely used by the society in Randa (Djibouti), it is worth subjecting these plants to investigation for the discovery of drugs with an anticipation of obtaining new bioactive substances. Randa with its unique climate and vegetation [1] may disclose some endemic species which have not been tested previously.

Poorly developed prevention system and resistance to drugs are the two main factors aggravating microbial infections in Djibouti [2-4]. This is related to the high prevalence in Djibouti of infectious diseases, probably caused by poverty and the general unhygienic conditions observed in many parts of Randa region.

After identifying traditional medicinal plants in Randa (Djibouti) by interviewing and conducting discussions with informants, priority diseases/disease categories were screened $[5,6]$.

Almost $20 \%$ of the medicinal plants recorded were those used by the people against infections [1]. This study aims at a selection of medicinal plants used for the care of cases of infectious diseases, and which have pharmacology properties.

To further strengthen the validity of the present work, a critical literature review was made followed by a simple comparison of the selected medicinal plants with the previous data gathered from the literature.

\section{MATERIALS AND METHODS}

As mentioned previously [1], the ethnobotanical study was conducted in Tadjourah District of Randa region in Djibouti, where 184 informants were interviewed from July 2010 to February 2011.
In this first study, the Informant Consensus Factor (ICF) was calculated for each ailment category to identify the agreements of the informants on the reported cures for the group of diseases $[7,8]$ and the Fidelity Level (FL') Index shows the percentage of informants claiming the use of a certain plant for the same ailment. We introduced additional statistical analysis for identifying candidate plants.

\section{Plant ratio (PR)}

Plant ratios towards a disease indicating the top ranking plants for a given disease, was calculated as $P R=(I p / I t) \times 100$, where $I p$ is the number of informants who independently indicated the use of a species for the same ailment and It is the total number of informants who mentioned any plant for the same disease. Ratios were rejected if $I p$ (the number of informants) that mentioned any plant for a particular disease is less than 15 in order to make certain that plants with higher consensus among community members are used for the analysis.

\section{Index of performance (Ip2) [9]}

To illustrate the selectivity of a plant for a specific ailment, a comparison was made between the expected and the observed values of the proportion of citation of a plant for a spefiic disease. The difference (P1-P2) between the two proportions was then used to define an Ip2 performance index which ranged from 0 to 3 according to an arbitrary scale rationalized for the purpose. The calculation P1-P2 was made as follows:

$$
\begin{aligned}
& \mathrm{P} 1=\mathrm{C} 1 / \mathrm{C} 2 \\
& \mathrm{P} 2=\mathrm{C} 3 / \mathrm{C} 4
\end{aligned}
$$

$\mathrm{C} 1$ = number of citations of a plant $\mathrm{A}$ for treating a specific disease $\mathrm{B}$, 
$\mathrm{C} 2=$ number of citations of the same plant $\mathrm{A}$ in the global list,

C3 $=$ total number of citations of the disease $B$,

$\mathrm{C} 4=\mathrm{a}$ total number of citations for all ailments.

If $0<\mathrm{P} 1-\mathrm{P} 2<1 / 3$, therefore $\mathrm{Ip} 2=3$, which denotes a very low performance.

If $1 / 3<\mathrm{P} 1-\mathrm{P} 2<2 / 3$, therefore Ip2 $=2$, which denotes an average performance.

If $\mathrm{P} 1-\mathrm{P} 2>2 / 3$, therefore Ip2 $=1$, which denotes a very high performance.

If $\mathrm{P} 1-\mathrm{P} 2<0$, therefore Ip2 $=0$, which denotes no performance.

\section{The informant consensus factor (ICF)}

The ICF was calculated for each ailment category, to identify the agreements of the informants on the reported cures for the group of diseases $[7,8]$. The Fidelity Level (FL') Index shows the percentage of informants claiming the use of a certain plant for the same ailment FL' ranking was taken into account only if the index of performance (Ip2) is $\leq 3$. The following scales were adopted for the purpose of comparing and prioritizing on the basis of the ranks of the different species: If FL' 0-33, Rank = 3: average performance; FL'34-66, Rank= 2: high performance; FL'>66, Rank = 1: very high performance. We introduced additional statistical analysis for identifying candidate plants.

\section{Preference ranking}

Preference ranking exercise was performed to assess the degree of effectiveness of certain medicinal plants against the most prevalent diseases in the area.

The data obtained were summarized through calculations of percentages and other values and presented in tables and fig. for further analysis and interpretation.

\section{RESULTS AND DISCUSSION}

\section{Identification of candidate plants by FL' ranking}

Fidelity Level (FL') was calculated for each Djiboutian medicinal plant. For each microbial ailment, each plant was ranked to find the appropriate candidate plant for the ailment. The results obtained are summarizing in table 1 .

Table 1: FL' values indicating the top ranking plants for a given disease*

\begin{tabular}{|c|c|c|c|c|c|c|c|c|c|}
\hline Scientific names & Local names & Diseases & Category & Number of uses & IP' & Iu & FL' & $\%$ & Rank \\
\hline Ochradenus baccatus Delile & malboyta & diphteria & Microbial & 16 & 16 & 16 & 100 & 100 & 1 \\
\hline Acacia tortilis (Forssk.) Hayne & eqebto & diphteria & Microbial & 3 & 3 & 8 & 37.5 & 38 & 2 \\
\hline Acacia seyal Delile & qadgento & dysentery & Microbial & 12 & 12 & 16 & 75 & 75 & 1 \\
\hline Ziziphus mauritiana Lam. & kusra & eye infection & Microbial & 5 & 5 & 28 & 17.8 & 18 & 3 \\
\hline Boscia coriacea Pax. & aytinaba & jaundice & Microbial & 4 & 4 & 10 & 40 & 40 & 2 \\
\hline Terminalia brownii Fresen. & wayboyta & jaundice & Microbial & 95 & 95 & 113 & 84.1 & 84 & 1 \\
\hline Balanites rotundifolia (Tiegh.) Blatt & alayto & parasitic worms & Microbial & 3 & 3 & 74 & 4.0 & 4 & 3 \\
\hline Orthosiphon pallidus Royle ex Benth. & abursaafiqi & parasitic worms & Microbial & 6 & 6 & 16 & 37.5 & 38 & 2 \\
\hline Melilotus suaveolens Ledeb. & meclab & poliomyelitis & Microbial & 3 & 3 & 6 & 50 & 50 & 2 \\
\hline
\end{tabular}

*(FL' was taken into account only if IP' $\geq 3$. The following scales were adopted for the purpose of comparing and prioritizing on the basis of the ranks of the different species: If FL' 0-33, Rank = 3: average performance; FL'34-66, Rank= 2: high performance; FL'>66, Rank = 1: very high performance). Malboyta (Ochradenus baccatus Delile) for diphtheria, Qadgento (Acacia seyal Delile) against dysentery and Wayboyta (Terminalia brownii Fresen.) against jaundice are the top species to study according to the fidelity level FL'.

\section{Identification of candidate plants by calculating plant ratios}

The plants that are most interesting for antimicrobial tests are the following 3 species, which had 15 or higher mentions of any plant and a reasonably good proportion (about half and higher) informant consensus [10-14].

It can be seen that those diseases that received 15 and above mentions of any medicinal plant can be priority species. A few species that could be considered in the first priority group include: Malboyta (Ochradenus baccatus) with 50\% reliability for use against diphtheria, Qadgento (Acacia seyal) with $80 \%$ reliability against dysentery, Wayboyta (Terminalia brownii) with 90\% reliability against jaundice and Abursaafiqi (Orthosiphon pallidus) with 40\% reliability against parasites. Likewise, the top three species of each category can be identified based on the ranks. Others plants species (including those that turned out receiving only one citation) could also be checked for their activities as long as resources are available, but it is noted that they were backed by minimal number of informants, notwithstanding the fact that even a plant mentioned by one person only could be effective for the particular disease. In other words, those plants with a reasonable number of citations can be compared amongst themselves rather than with those that were cited by few informants.

Identification of microbial candidate plants by calculating Index performance (IP2)

The Index of the performance of the microbial infection is presented in table 3. Galqaddo (Tarchonanthus camphoratus), Malboyta (Ochradenus baccatus) against diphtheria had an IP2 value of 0.97 , 0.73 for Qadgento (Acacia seyal) against dysentery and finally Dataamqada (Heliotropium longiflorum) for infections.

\section{Comparison of the different factors}

Plant species that are widely used as remedies for treating microbial infections were compared using FL' (Fidelity Level), IP2 and PR ranking; and by judging the combined effectiveness. The species that ranked top and selected as priority candidates for testing are shown in table 5. Each of the 18 species in table 4, can be considered as good enough candidates for pharmacological testing for the diseases under which they are listed.

The FL' values of medicinal plants cited by three or more informants, for being used against the antimicrobial category were compared, and nine of them were selected. These nine plant species with high FL' values also had high PR ratios towards a given disease. Among them, the anti-dysentery claim for Qadgento (Acacia seyal) deserves special attention in the context of increasing the success rate in the search for anti-viral drugs. Another interesting finding is the use of Eqebto (Acacia tortillis) and Malboyta (Ochradenus baccatus) for diphtheria.

Other new information found in this analysis is the use of Aytinaba (Boscia coriacea) and Wayboyta (Terminilia brownii) as anti-jaundice agents. Despite their moderate average rank 3, 11 others plants species were also selected to be studied. Those 11 plants had already a selection criterion of 15 for plant ratios and were chosen to be included in the study.

A bibliographical research on the pharmacological properties and traditional uses was made [15-106]. The citations number was obtained by adding them for every plant. These data allow to define the degree of knowledge and to guide possible studies on medicinal plants cited in table 5 . 
Table 2: Plant ratios indicating the top ranking plants for a given disease*

\begin{tabular}{|c|c|c|c|c|c|c|}
\hline Scientific names & Plant & Disease & $\begin{array}{l}\text { Number of } \\
\text { informants } \\
\text { (Ip) }\end{array}$ & $\begin{array}{l}\text { Number of informants } \\
\text { who mentioned any plant } \\
\text { for the same disease (It) }\end{array}$ & $\begin{array}{l}\text { Plants ratio } \\
\text { towards a } \\
\text { disease }\end{array}$ & $\begin{array}{l}\text { Plant } \\
\text { rank }\end{array}$ \\
\hline Ochradenus baccatus Delile. & malboyta & diphteria & 16 & 32 & 50 & 1 \\
\hline $\begin{array}{l}\text { Acacia tortilis (Forssk.) } \\
\text { Hayne. }\end{array}$ & eqebto & & 3 & 32 & 9 & 2 \\
\hline Acalypha fruticosa Forssk. & darmuusa & & 2 & 32 & 6 & 3 \\
\hline Grewia erythreae Schweinf. & cedayto & & 2 & 32 & 6 & 3 \\
\hline Melilotus suaveolens Ledeb. & meclab & & 2 & 32 & 6 & 3 \\
\hline $\begin{array}{l}\text { Indigofera oblongifolia } \\
\text { Forssk. }\end{array}$ & $\begin{array}{l}\text { xacanle } \\
\text { baro-barbaré }\end{array}$ & & 1 & 32 & 3 & 6 \\
\hline Ruta chalepensis L. & sidaaba & & 1 & 32 & 3 & 6 \\
\hline Capparis cartilaginea Decne. & xaxaq laynota & & 1 & 32 & 3 & 6 \\
\hline Tarchonanthus camphoratus L. & galqaddo & & 1 & 32 & 3 & 6 \\
\hline Maerua triphylla A. Rich. & ruqaysi & & 1 & 32 & 3 & 6 \\
\hline Aristolochia bracteolata Lam. & suqsuuqi & & 1 & 32 & 3 & 6 \\
\hline Acacia seyal Delile. & qadgento & & 12 & 15 & 80 & 1 \\
\hline Ziziphus mauritiana L. & kusra & dysentery & 2 & 15 & 13 & 2 \\
\hline Solanum somalense Franch. & garbaqaddoyta & & 1 & 15 & 7 & 3 \\
\hline Terminalia brownii Fresen. & wayboyta & & 95 & 105 & 90 & 1 \\
\hline Boscia coriacea Pax. & aytinaba & & 4 & 105 & 4 & 2 \\
\hline $\begin{array}{l}\text { Balanites rotundifolia } \\
\text { (Tiegh.) Blatt. }\end{array}$ & alayto & jaundice & 2 & 105 & 2 & 4 \\
\hline $\begin{array}{l}\text { Cymbopogon commutatus } \\
\text { (Steud.) Stapf. }\end{array}$ & dambahu & & 1 & 105 & 1 & 5 \\
\hline $\begin{array}{l}\text { Orthosiphon pallidus Royle } \\
\text { ex Benth. }\end{array}$ & abursaafiqi & & 6 & 15 & 40 & 1 \\
\hline $\begin{array}{l}\text { Balanites rotundifolia } \\
\text { (Tiegh.) Blatt. }\end{array}$ & alayto & & 3 & 15 & 20 & 2 \\
\hline Terminalia brownii Fresen. & wayboyta & parasite & 2 & 15 & 13 & 3 \\
\hline Buxus hildebrandtii Baill. & gaydarto & & 1 & 15 & 7 & 4 \\
\hline Ocimum basilicum L. & laynoyta & & 1 & 15 & 7 & 4 \\
\hline
\end{tabular}

*Ratios are rejected if Ip the number of informants that mentioned any plant for a particular disease is less than 15.

Table 3: Index of performance (IP2) of the plants from randa region within the microbial infection*

\begin{tabular}{|c|c|c|c|c|c|c|c|c|c|c|c|}
\hline $\begin{array}{l}\text { Local } \\
\text { names }\end{array}$ & Scientific names & $\begin{array}{l}\text { Disease } \\
\text { name }\end{array}$ & $\begin{array}{l}\text { Number of } \\
\text { informants }\end{array}$ & C1 & $\mathrm{C} 2$ & C3 & C4 & P1 & P2 & D & $\begin{array}{l}\text { IP } \\
2\end{array}$ \\
\hline $\begin{array}{l}\text { Xaxaq } \\
\text { Laynoyta }\end{array}$ & Capparis cartilaginea Decne. & Dipheteria & 1 & 1 & 2 & 32 & 920 & 0.50 & 0.03 & 0.47 & 2 \\
\hline Darmuusa & Acalypha fruticosa Forssk. & Dipheteria & 2 & 0 & 24 & 32 & 920 & 0.08 & 0.03 & 0.05 & 3 \\
\hline Eqebto & Acacia tortillis (Forssk.) Hayne. & Dipheteria & 3 & 3 & 8 & 32 & 920 & 0.38 & 0.03 & 0.34 & 2 \\
\hline Galqaddo & Tarchonanthus camphoratus L. & Dipheteria & 1 & 1 & 1 & 32 & 920 & 1.00 & 0.03 & 0.97 & 1 \\
\hline Cedayto & Grewia erythraea Schweinf. & Dipheterie & 2 & 2 & 14 & 32 & 920 & 0.14 & 0.03 & 0.11 & 3 \\
\hline Ruqaysi & Maerua triphylla A. Rich. & Diphteria & 1 & 1 & 8 & 32 & 920 & 0.13 & 0.03 & 0.09 & 3 \\
\hline Sidaaba & Ruta chalepensis L. & Diphteria & 1 & 1 & 27 & 32 & 920 & 0.04 & 0.03 & 0.00 & 3 \\
\hline Suqsuuqi & Aristolochia bracteolata Lam. & Diphteria & 1 & 1 & 42 & 32 & 920 & 0.02 & 0.03 & -0.01 & 0 \\
\hline $\begin{array}{l}\text { Xacanle } \\
\text { Baro- } \\
\text { babaré }\end{array}$ & Indigofera oblongifolia Forssk. & Diphtheria & 1 & 1 & 3 & 32 & 920 & 0.33 & 0.03 & 0.30 & 3 \\
\hline Malboyta & Ochradenus baccatus Delile. & Diphtheria & 16 & 16 & 16 & 32 & 920 & 1.00 & 0.03 & 0.97 & 1 \\
\hline Meclab & Melilotus suaveolens Ledeb. & Diphtheria & 2 & 0 & 6 & 32 & 920 & 0.33 & 0.03 & 0.30 & 3 \\
\hline $\begin{array}{l}\text { Garbaqadd } \\
\text { oyta }\end{array}$ & Solanum somalense Franch. & Dysentery & 1 & 1 & 11 & 15 & 920 & 0.09 & 0.02 & 0.07 & 3 \\
\hline Kusra & Ziziphus mauritiana L. & Dysentery & 2 & 7 & 20 & 15 & 920 & 0.10 & 0.02 & 0.08 & 3 \\
\hline Qadgento & Acacia seyal Delile. & Dysentery & 12 & 12 & 16 & 15 & 920 & 0.75 & 0.02 & 0.73 & 1 \\
\hline Kusra & Ziziphus mauritiana L. & Eye infection & 5 & 0 & 28 & 11 & 920 & 0.18 & 0.01 & 0.17 & 3 \\
\hline $\begin{array}{l}\text { Data- } \\
\text { amqada }\end{array}$ & $\begin{array}{l}\text { Heliotropium longiflorum (A. DC.). } \\
\text { Jaub and Sapch. }\end{array}$ & Infection & 1 & 1 & 2 & 8 & 920 & 0.50 & 0.01 & 0.49 & 2 \\
\hline $\begin{array}{l}\text { Gaydarceel } \\
\text { a }\end{array}$ & Dodonaea angustifolia L. f. & Infection & 1 & 1 & 5 & 8 & 920 & 0.20 & 0.01 & 0.19 & 3 \\
\hline Kurbeyta & $\begin{array}{l}\text { Commiphora erythraea (Ehrenb.) } \\
\text { Engl. }\end{array}$ & Infection & 1 & 1 & 8 & 8 & 920 & 0.13 & 0.01 & 0.12 & 3 \\
\hline Darmuusa & Acalypha fruticosa Forssk. & Infection & 2 & 4 & 24 & 8 & 920 & 0.08 & 0.01 & 0.07 & 3 \\
\hline Dambahu & $\begin{array}{l}\text { Cymbopogon commutatus } \\
\text { (Steud.) } \\
\text { and Stapf. }\end{array}$ & Jaundice & 1 & 1 & 14 & 105 & 920 & 0.07 & 0.11 & -0.04 & 0 \\
\hline Alayto & $\begin{array}{l}\text { Balanites rotundifolia (Tiegh.) } \\
\text { Blatt. }\end{array}$ & Jaundice & 2 & 6 & 74 & 105 & 920 & 0.03 & 0.11 & -0.09 & 0 \\
\hline Aytinaba & Boscia coriacea Pax. & Jaundice & 4 & 4 & 10 & 105 & 920 & 0.40 & 0.11 & 0.29 & 3 \\
\hline Wayboyta & Terminalia brownii Fresen. & Jaundice & 95 & 97 & 11 & 105 & 920 & 0.84 & 0.11 & 0.73 & 1 \\
\hline
\end{tabular}




\begin{tabular}{|c|c|c|c|c|c|c|c|c|c|c|c|}
\hline & & & & & 3 & & & & & & \\
\hline Alayto & $\begin{array}{l}\text { Balanites rotundifolia (Tiegh.) } \\
\text { Blatt. }\end{array}$ & Malaria & 1 & 0 & 74 & 1 & 920 & 0.01 & 0.00 & 0.01 & 3 \\
\hline $\begin{array}{l}\text { Abursaafiq } \\
\text { i }\end{array}$ & $\begin{array}{l}\text { Orthosiphon pallidus Royle ex } \\
\text { Benth. }\end{array}$ & Parasite & 6 & 6 & 16 & 15 & 920 & 0.38 & 0.02 & 0.36 & 2 \\
\hline Gaydarto & Buxus hidelbrandtii Baill. & Parasite & 1 & 1 & 3 & 15 & 920 & 0.33 & 0.02 & 0.32 & 3 \\
\hline Laynota & Ocimum basilicum L. & Parasite & 1 & 1 & 1 & 15 & 920 & 1.00 & 0.02 & 0.98 & 1 \\
\hline Alayto & $\begin{array}{l}\text { Balanites rotundifolia (Tiegh.) } \\
\text { Blatt. }\end{array}$ & Parasite & 3 & 0 & 74 & 15 & 920 & 0.04 & 0.02 & 0.02 & 3 \\
\hline Wayboyta & Terminalia brownii Fresen. & Parasite & 2 & 0 & $\begin{array}{l}11 \\
3\end{array}$ & 15 & 920 & 0.02 & 0.02 & 0.00 & 3 \\
\hline Meclab & Melilotus suaveolens Ledeb. & Polio & 3 & 5 & 6 & 3 & 920 & 0.50 & 0.00 & 0.50 & 2 \\
\hline Qubaabulto & Withania somnifera (L.) Dunal. & Tuberculosis & 1 & 1 & 10 & 4 & 920 & 0.10 & 0.00 & 0.10 & 3 \\
\hline Udda & Balanites aegyptiaca (L.) Delile. & Tuberculosis & 2 & 2 & 7 & 4 & 920 & 0.29 & 0.00 & 0.28 & 3 \\
\hline Ayrobeya & Indigofera articulata Gouan. & Tuberculosis & 1 & 1 & 9 & 4 & 920 & 0.11 & 0.00 & 0.11 & 3 \\
\hline
\end{tabular}

$*$ If $\mathrm{D}<0$, IP2 = 4: below average performance; if $0<\mathrm{D}<0.33$ IP2=3: average performance; $0.34<\mathrm{D}<0.67$ IP2 $=2$, high performance, If $\mathrm{D}>0.68$ IP2 $=1$, very high performance.

Table 4: Important species in their rank order

\begin{tabular}{|c|c|c|c|c|c|c|}
\hline \multirow{2}{*}{$\begin{array}{l}\text { Local name of } \\
\text { disease }\end{array}$} & \multirow[t]{2}{*}{ Disease category } & \multirow[t]{2}{*}{ Local name of plant } & \multirow[t]{2}{*}{ Scientific name } & \multicolumn{3}{|c|}{ Rank } \\
\hline & & & & FL' & IP2 & PR \\
\hline \multirow[t]{6}{*}{ Diphtheria } & \multirow[t]{6}{*}{ Microbial Infection } & Malboyta & Ochradenus baccatus Delile. & 1 & 1 & 1 \\
\hline & & meclab & Melilotus suaveolens Ledeb. & $\mathrm{ND}^{*}$ & 3 & 3 \\
\hline & & eqebto & Acacia tortilis (Forssk.) Hayne. & 2 & 2 & 2 \\
\hline & & darmussa & Acalypha fruticosa Forssk. & ND & 3 & 3 \\
\hline & & cedayto & Grewia erythraea Schweinf. & ND & 3 & 3 \\
\hline & & galqaddo & Tarchonanthus camphoratus L. & ND & 1 & ND \\
\hline \multirow[t]{3}{*}{ Dysentery } & \multirow[t]{3}{*}{ Microbial infection } & Qadgento & Acacia seyal Delile. & 1 & 1 & 1 \\
\hline & & kusra & Ziziphus mauritiana L. & ND & 3 & 2 \\
\hline & & garbaqaddoyta & Solanum somalense Franch. & ND & 3 & 3 \\
\hline \multirow[t]{2}{*}{ Jaundice } & \multirow[t]{2}{*}{ Microbial infection } & Wayboyta & Terminalia brownii Fresen. & 1 & 1 & 1 \\
\hline & & aytinaba & Boscia coriacea Pax. & 2 & 3 & 2 \\
\hline \multirow[t]{3}{*}{ Parasitic worms } & \multirow[t]{3}{*}{ Microbial infection } & Wayboyta & Terminalia brownii Fresen. & ND & 3 & 3 \\
\hline & & abursaafiqi & Orthosiphon pallidus Royle ex Benth. & 2 & 2 & 1 \\
\hline & & alayto & Balanites rotundifolia (Tiegh.) Blatt. & 3 & 3 & 2 \\
\hline Malaria & Microbial infection & Alayto & Balanites rotundifolia (Tiegh.) Blatt. & ND & 3 & ND \\
\hline \multirow[t]{3}{*}{ Tuberculosis } & \multirow[t]{3}{*}{ Microbial infection } & Qubaabulta & Withania somnifera (L.) Dunal. & ND & 3 & ND \\
\hline & & udda & Balanites aegyptiaca (L.) Delile. & ND & 3 & ND \\
\hline & & ayrobeya & Indigofera articulata Gouan. & ND & 3 & ND \\
\hline \multirow{5}{*}{$\begin{array}{l}\text { Other microbial } \\
\text { infections }\end{array}$} & \multirow{5}{*}{$\begin{array}{l}\text { Other microbial } \\
\text { infections }\end{array}$} & Kusra & Ziziphus mauritiana L. & 1 & 3 & ND \\
\hline & & darmussa & Acalypha fruticosa Forssk. & ND & 3 & ND \\
\hline & & gaydarceela & Dodonea angustifolia L. f. & ND & 2 & ND \\
\hline & & kurbeyta & Commiphora erythraea (Ehrenb.) Engl. & ND & 3 & ND \\
\hline & & Data-amqada & $\begin{array}{l}\text { Heliotropium longiflorum (A. DC.) Jaub. and } \\
\text { Spach. }\end{array}$ & ND & 2 & ND \\
\hline
\end{tabular}

*ND represents values that were out of the range.

Table 5: Comparison of information on medicinal plant species found in randa region, djibouti with the literature

\begin{tabular}{|c|c|c|c|c|}
\hline \multirow[t]{2}{*}{ Most cited species } & \multirow[t]{2}{*}{ Local name } & \multicolumn{3}{|l|}{ Literature found in } \\
\hline & & Pharmacological & Traditional medicine & $\begin{array}{l}\text { Number of } \\
\text { citations }\end{array}$ \\
\hline Acacia tortilis (Forssk.) Hayne. & eqebto & {$[15-22]$} & {$[15,17,18,22]$} & $8+4=12$ \\
\hline Acacia seyal Delile. & qadgento & [23-25] & {$[23,24,26-28]$} & $3+5=8$ \\
\hline Terminalia brownie Fresen. & wayboyta & {$[29,30]$} & {$[31,30]$} & $2+2=4$ \\
\hline Ochradenus baccatus Delile. & malboyta & {$[32-34]$} & [35] & $3+1=4$ \\
\hline Boscia coriacea Pax. & aytinaba & [36] & [37-39] & $1+3=4$ \\
\hline Melilotus suaveolens Lebed. & meclab & [40] & [41-42] & $1+2=3$ \\
\hline Balanites rotundifolia (Tiegh.) Blatt. & alayto & & {$[43-44]$} & $0+2=2$ \\
\hline Ziziphus mauritiana L. & kusra & {$[45-47,43,48]$} & {$[48-50,44,51-54]$} & $5+8=13$ \\
\hline Orthosiphon pallidus Royle ex Benth. & abursaafiqi & {$[55]$} & {$[55]$} & $1+1$ \\
\hline Acalypha fruticosa Forssk. & darmussa & {$[56-58]$} & {$[6,14,37,40,45,54,56,66]$} & $3+15=18$ \\
\hline Grewia erythraea Schweinf. & cedayto & [66] & {$[66,67]$} & $1+2=3$ \\
\hline Solanum somalense Franch. & Garbaaqaddoyta & [68] & {$[14,68]$} & $1+2=3$ \\
\hline Withania somnifera (L.) Dunal. & Qubaabulto & [69-76] & {$[71,72,77-79]$} & $8+5=13$ \\
\hline Balanites aegyptiaca (L.) Delile. & udda & [80-89] & {$[62,81,82,90-95]$} & $10+10=20$ \\
\hline Indigofera articulata Gouan. & ayrobeya & & & 0 \\
\hline Dodonaea angustifolia $\mathrm{L} . \mathrm{f}$. & gaydarcela & {$[96-98]$} & {$[96,99-103]$} & $3+6=9$ \\
\hline Heliotropium longiflorum (A. DC.) Jaub. and Spach. & data amqada & & {$[104]$} & $0+1=1$ \\
\hline Comniphora erythraea (Ehrenb.) Engl. & kurbeyta & {$[79,105,106]$} & {$[79,105]$} & $3+2=5$ \\
\hline
\end{tabular}




\section{CONCLUSION}

By simple comparisons of the different factors (IP2, PR, and FL') of plant screening, 18 plants species were selected to be the most potent with good healing results. Most of these recorded plants in our study of Randa region of Djibouti were also used in other countries where traditional medicine still contributes to healthcare services. This indicates the medicinal potential of these plants and their ability to have curative properties. Few medicinal plants used in Djibouti as having antimicrobial properties were not found in the literature. The first selected plants to be further involved in scientific studies would be those which have not been tested before against bacteria. The list included: Meclab (Melilotus suaveolens), Alayto (Balanites rotundifolia), Abursaafiqi (Orthosiphon pallidus), Garbaqaddoyta (Solanum somalense), Ayrobeya (Indigofera articulata) and Data-amqada (Heliotropium longiflorum). Confirmation of the data by simple bioassay analysis and further on by isolation of the active principles could lead to templates to the synthesis of modern drugs.

\section{CONFLICTS OF INTERESTS}

\section{Declared none}

\section{REFERENCES}

1. Hassan Abdallah A, Merito A, Hassan S, Aboubaker D, Djama M, Asfaw Z, et al. Medicinal plants and their uses by the people in the region of randa, djibouti. J Ethnopharmacol 2013;148:701-13.

2. WHO, Djibouti Tuberculosis profile; 2010. Available from: http://www.who.int/tb/data. [Last accessed on $10 \mathrm{Feb} 2015]$.

3. WHO, Stop Tuberculosis Programme; 2009. Available from: http://www.emro.who.int/djibouti. [Last accessed on $10 \mathrm{Feb}$ 2015].

4. WHO; 2009. Available from: www.who.int/malaria/ publications/ country-profiles/profile_dji_pdf. [Last accessed on $10 \mathrm{Feb}$ 2015].

5. Martin GJ. Ethnobotany: a 'people and plants' conservation manual. Chapman and Hall, London; 268. p. 1995.

6. Cotton CM. Ethnobotany: Principles and Applications. John Wiley, Sons, New York; 1996. p. 424.

7. Heinrich M. Ethnobotany and its role in drug development. Phytother Res 2000;14:479-88.

8. Heinrich M, Ankli A, Frei B, Weimann C, Sticher O. Medicinal plants in Mexico: healers consensus and cultural importance. Soc Sci Med 1998;47:1863-75.

9. Betti JL. An ethnobotanical study of medicinal plants among the baka pygmies in the dja biosphere reserve, Cameroon African. Afr Study Monographs 2004;25:1-27.

10. Alexiades MN. Selected guidelines for ethnobotanical research: a field manual. Advances in economic botany. Vol. 10. The New York Botanical Garden, Bronx; 1996.

11. Friedman J, Yaniv Z, Dafni A, Palewitch D. A preliminary classification of the healing potential of medicinal plants, based on the rational analysis of an ethnopharmacological field survey among bedouins in negev desert, Israel. J Ethnopharmacol 1986;16:275-87.

12. Robleh YD. Rapporteur national: rapport national sur L'etat des ressources phytogenetiques. Pour L'Alimentation Et L'Agriculture; 2007. p. 1-63.

13. Audru J, Cesar J, Cesar J, Lebrun JP. Flore illustree: les plantes vasculaires de la republique de djibouti, mission française et de cooperation culturelle de djibouti. Cirad-EMVT 1994;1:1-300.

14. Lefloc'h E, Lemordant D, Lignon A, Rezkallah N. Pratiques ethnobotaniques des populations afars de la moyenne vallée d'Awash (Ethiopie). J Ethnopharmacol 1985;14:283-314.

15. Al-Fatimi M, Wurster M, Schroder G, Lindequist. Antioxidant, antimicrobial and cytotoxic activities of selected medicinal plants from Yemen. J Ethnopharmacol 2007;111:657-66.

16. Maregesi SM, Pieters L, Ngassapa OD, Apers S, Vingerhoets R, Cos $\mathrm{P}$, et al. Screening of some tanzanian medicinal plants from Bunda district for antibacterial, antifungal and antiviral activities. J Ethnopharmacol 2008;119:58-66.

17. Saini ML, Saini R, Roy S, Kumar A. Comparative pharmacognostical and antimicrobial studies of acacia species (Mimosaceae). J Med Plants Res 2008;2:378-86.
18. Kubmarawa D, Ajoku GA, Enwerem NM, Okorie DA. Preliminary phytochemical and antimicrobial screening of 50 medicinal plants from Nigeria. Afr J Biotechnol 2007;6:1690-6.

19. Kigondua EVM, Rukunga GM, Kerikoc JM, Tonui WK, Gathirwab JW, Kirirab PG, et al. Antiparasitic activity and cytotoxicity of selected medicinal plants from Kenya. J Ethnopharmacol 2009;123:504-9.

20. Samuelsson G, Farah MH, Claeson P, Hagos M, Thulin M, Hedberg 0 , et al. Inventory of plants used in traditional medicine in somalia. Plants of the families lauraceaepapilionaceae. J Ethnopharmacol 1992;37:93-112.

21. Pillay $P$, Maharaj VJ. Investigating South African plants as a source of new antimalarial drugs. J Ethnopharmacol 2008;119:438-54.

22. Koch A, Tamez P, Pezzuto J, Soejarto D. Evaluation of plants used for antimalarial treatment by the Maasai of Kenya. J Ethnopharmacol 2005;101:95-9.

23. Eldeen IMS, Van Staden J. Cyclooxygenase inhibition and antimycobacterial effects of extracts from sudanese medicinal plants. S Afr J Bot 2008;74:225-9.

24. Eldeen IMS, Van Staden J. In vitro pharmacological investigation of extracts from some trees used in sudanese traditional medicine. S Afr J Bot 2007;73:435-40.

25. McGawa LJ, Lall N, Meyerb JJM, Eloff JN. The potential of South African plants against mycobacterium infection. J Ethnopharmacol 2008;119:482-500.

26. Ngutaa M, Mbariaa JM, Gakuyab DW, Gathumbi PK, Kiamad SG. Traditional antimalarial phytotherapy remedies used by the South Coast community, Kenya. J Ethnopharmacol 2010; 131:256-67.

27. Salem AZM, Robinson PH, El-Adawy MM, Hassan AA. In vitro fermentation and microbial protein synthesis of some browse, tree leaves with or without the addition of polyethylene glycol. Anim Feed Sci Technol 2007;138:318-30.

28. Geissler PW, Harris ST, Prince RJ, Olsen A, Odhiambo RA, Oketch-Rabah $\mathrm{H}$, et al. Medicinal plants used by Luo mothers and children in Bondo district, Kenya. J Ethnopharmacol 2002;83:39-54.

29. Mbwambo ZH, Moshi MJ, Masimba PJ, Kapingu MC, SO Nondo R. Antimicrobial activity and brine shrimp toxicity of extracts of Terminalia brownii roots and stem. BMC Complementary Altern Med 2007;7:9.

30. Kareru PG, Gachanja AN, Keriko JM, Kenji GM. Antimicrobial activity of some medicinal plants used by herbalists in Eastern Province, Kenya. Afr J Tradit Complementary Altern Med 2008;5:51-5.

31. Kareru PG, Kenji GM, Gachanja AN, Keriko JM, Mungai G. Traditional medicines among the Embu and Mbeere peoples of Kenya. Afr J Tradit Complementary Altern Med 2007;4:75-86.

32. Abutbul S, Golan-Goldhirsh A, Barazani O, Ofir R, Zilberg D. Screening of desert plants for use against bacterial pathogens in fish. Israeli J Aquaculture Bamidgeh 2005;57:71-80.

33. Abdel Sattar E, Harraz FM, El Gayed SH. Antimicrobial activity of extracts of some plants collected from the kingdom of Saudi Arabia. Med Sci 2008;15:25-33.

34. Barakat HH, El-Mousallamy AMD, Souleman AMA, Awadalla S. Flavonoids of Ochradenus baccatus. Phytochemistry 1991;30:3777-9.

35. El-Ghazali GE, Al-Khalifa KS, Saleem GA, Abdallah EM. Traditional medicinal plants are indigenous to Al-Rass province, Saudi Arabia. J Med Plants Res 2010;4:2680-3.

36. Kiswii TM. Aspergillus flavus and aflatoxin levels in stored maize in Eastern kenya and antifungal activity of some plant extracts department: plant and Microbial Sciences. Thesis. Available from: http://hdl.handle.net/123456789/393;2011. [Last accessed on 10 Mar 2015].

37. Ismail MYM, Assem NM, Zakriya M. Botanicals promoting oral and dental hygiene: a review. Res J Pharm Biol Chem Sci 2010;1:202-6.

38. Loewenthal R, Pe'er J. Traditional methods used in the treatment of ophthalmic diseases among the turkana tribe in North West Kenya. J Ethnopharmacol 1991;33:227-9.

39. Teklehaymanot T, Giday M. Quantitative ethnobotany of medicinal plants used by Kara and Kwego semi-pastoralist 
people in lower omo river valley, debub omo zone, southern nations, nationalities and peoples Regional State, Ethiopia. J Ethnopharmacol 2010;130:76-84.

40. Lei Zhao, Jun-Yan Tao, Shu-Ling Zhang, Ran Pang, Feng Jin, JiHua Dong, et al. Inner anti-inflammatory mechanisms of petroleum ether extract from Melilotus suaveolens Ledeb. Inflammation 2007;30:213-23.

41. Moskalenko SA. Slavic ethnomedicine in the soviet far east part I: herbal remedies among Russians/Ukrainians in the sukhodol valley, primorye. J Ethnopharmacol 1987;21:231-51.

42. Dung NXD, Loi DT. Selection of traditional medicines for study. J Ethnopharmacol 1991;32:57-70.

43. Khairul Ikram EH, Khoo Hock Eng K, Ismail AMMJA, Idris S, Azlan A, et al. Antioxidant capacity and total phenolic content of Malaysian underutilized fruits. J Food Compos Anal 2009;22:388-93.

44. Inngjerdingen $\mathrm{K}$, Nergård $\mathrm{CS}$, Diallo $\mathrm{D}$, Mounkoro $\mathrm{PP}$, Berit Smestad Paulsen BS. An ethnopharmacological survey of plants used for wound healing in dogonland, Mali, West Africa. J Ethnopharmacol 2004;92:233-44.

45. Adamu HM, Abayeh OJ, Agho MO, Abdullahi AL, Uba A, Dukku $\mathrm{HU}$, et al. An ethnobotanical survey of bauchi state herbal plants and their antimicrobial activity. J Ethnopharmacol 2005;99:1-4.

46. Deeni YY, Sadiq NM. Antimicrobial properties and phytochemical constituents of the leaves of African mistletoe (Tapinanthus dodoneifolius (DC) Danser) (Loranthaceae): an ethnomedicinal plant of Hausaland, Northern Nigeria. J Ethnopharmacol 2002;83:235-40.

47. Jain A, Katewa SS, Galav PK, Sharma P. Medicinal plant diversity of Sitamata wildlife Sanctuary, Rajasthan, India. J Ethnopharmacol 2005;102:143-57.

48. Tetalia P, Waghchaurea C, Daswanib PG, Antiab NH, Birdib TJ. Ethnobotanical survey of antidiarrhoeal plants of parinche valley, Pune district, Maharashtra, India. J Ethnopharmacol 2009;123:229-36.

49. Ghimire K, Bastakoti RR. Ethnomedicinal knowledge and healthcare practices among the Tharus of Nawalparasi district in central Nepal. For Ecol Manage 2009;257:2066-72.

50. Diehl MS, Atindehoub KK, Téré H, Betschart B. Prospect for anthelminthic plants in the Ivory Coast using ethnobotanical criteria. J Ethnopharmacol 2004;95:277-84.

51. Behera SK, Misra MK. Indigenous phytotherapy for genitourinary diseases used by the Kandha tribe of Orissa, India. J Ethnopharmacol 2005;102:319-25.

52. Mohagheghzadeh A, Faridi P, Shams-Ardakani M, Ghasemi YM. Medicinal smokes. J Ethnopharmacol 2006;108:161-84.

53. Hedberg I, Hedberg O, Madatic PJ, Mshigeni KE, Mshiif EN Samuelsson G. Inventory of plants used in traditional medicine in Tanzania. II. Plants of the families dilleniaceae-opiliaceae. J Ethnopharmacol 1983;9:105-28.

54. Watt JM, Brandwijk BMG. Medicinal and poisonous plants of Southern and Eastern Africa. $2^{\text {nd }}$ ed. E. and S. Livingstone Ltd., Edinburgh, London; 1962.

55. Ashokan K, Muthuraman MS. Anticancer studies on Orthosiphon pallidus royle and Peristrophe bicalyculata nees. J Pharm Res 2011;4:2654-6.

56. Hamza OJM, Carolien JP, van den Bout-van den Beukel, Mecky IN Matee, Moshi MJ, Frans HM, et al. Antifungal activity of some Tanzanian plants used traditionally for the treatment of fungal infections. J Ethnopharmacol 2006;108:124-32.

57. Alasbahi RH, Sfiyeva S, Craker LE. Antimicrobial activity of some yemen medicinal plants. J Herbs Spices Med Plants 1999;6:75-83.

58. Khan MR, Ndaalio G, Nkunya MHH, Wevers H, Sawhney AN. Studies on African medicinal plants. Part I. preliminary screening of medicinal plants for antibacterial activity. Planta Med 1980;40:91-7.

59. Nanyingi MO, Mbaria JM, Lanyasunya AL, GWagate C, Koros KB, Kaburia HF, et al. Ethnopharmacological survey of Samburu district, Kenya. J Ethnobiol Ethnomed 2008;4:14.

60. Rajkumar V, Guha G, Kumar RA. Therapeutic potential of Acalypha fruticosa. Food Chem Toxicol 2010;48:1709-13.
61. Chhabra SC, Mahunnah RLA, Mshiu EN. Plants used in traditional medicine in Eastern Tanzania. III. Angiosperms (Euphorbiaceae to Menispermaceae). J Ethnopharmacol 1990;28:255-83.

62. Mohagheghzadeh A, Mohammadreza PF, Ardakani S, Ghasemi Y. Medicinal smokes. J Ethnopharmacol 2006;108:161-84.

63. Kokwaro 0. Medicinal plants of East Africa, East African Literature Bureau, Kampala, Nairobi, Dar es Salaam; 1976.

64. Samuelsson G, Farah MH, Claesona P, Hagosa M, Thulinb M, Hedbersb 0 , et al. Inventory of plants used in traditional medicine in somalia. I. plants of the families acanthaceaechenopodiaceae. J Ethnopharmacol 1991;35:25-63.

65. Raza M, Choudhary MI, Atta-Ur-Rahman. Medicinal plants with anticonvulsant activities. Atta-ur-Rahman (Ed.). Stud Nat Prod Chem 2000;22:507-53.

66. Zaidi MA, Crow SA. Biologically active traditional medicinal herbs from Balochistan, Pakistan. J Ethnopharmacol 2005;96:331-4.

67. Zaidi MI, Crow SA. Biologically active medicinal plants of Pakistan. Res J Univ Balochistan 2003;2:24-8.

68. Seifu T. Ethnobotanical and ethnopharmaceutical studies on medicinal plants of Chifra district, Afar Region, North Eastern Ethiopia, Addis Ababa University School of Graduate Studies; 2004.

69. Owais M, Sharad KS, Shehbaz A, Saleemuddin M. Antibacterial efficacy of Withania somnifera (ashwagandha) an indigenous medicinal plant against experimental murines salmonellosis. Phytomedicine 2005;12:229-35.

70. Agarwal R, Diwanay S, Patki P, Patwardhan B. Studies on immunomodulatory activity of Withania somnifera (ashwagandha) extracts in experimental immune inflammation. J Ethanopharmocol 1999;67:27-35.

71. Weiner MA, Weiner J. Ashwagandha (Indian Ginseng) In: Herbs that Heal, Quantum Books, Mill Valley, California; 1994. p. 70-2.

72. Asthana R, Raina MK. Pharmacology of Withania somnifera. Indian Drugs 1989;26:199-204.

73. Elsakk M, Grigorescu E, Stanescu, Dorneanu V. New data referring to the chemistry of Whitania somnifera species. Rev Med Chir Soc Med Nat Lasi 1990;94:385-7.

74. Aldor Y, Walach N, Modai D, Horn Y. Zinc and copper levels in plasma erythrocytes and whole blood in cancer patients. Klin Wochenschr 1982;60:375-7.

75. Puri HS, Rasayana. Ayurvedic herbs for longevity and rejuvenation CRC Press: New York; 2002. p. 46-50.

76. Sinha S, Nos'al'ov'a G, Bandyopadhyay SS, Fle`skov'a D, Ray B. In vivo anti-tussive activity and structural features of a polysaccharide fraction from water extracted Withania somnifera. J Ethnopharmacol 2011;134:510-3.

77. Mathur R, Gupta SK, Singh N, Mathur S, Kochupillai Velpandian $\mathrm{T}$. Traditional plant knowledge and use by local healers in Sekory district Jimma Zone South Western Ethiopia. J Ethnopharmacol 2006;105:336-41.

78. Sudhir S, Budhiraja RD, Miglani GP, Arora B, Gupta IC, Gorg KN. Pharmacological studies on leaves of Withania somnifera. Planta Med 1986;1:61-3.

79. Al-Fatimi M, Wurster M, Schroder G, Lindequist U. Antioxidant, antimicrobial and cytotoxic activities of selected medicinal plants from Yemen. J Ethnopharmacol 2007;111:657-66.

80. Archibald RG. The use of the fruit of the tree Balanites aegyptica in control of schistosomiasis in the Sudan. Trans $\mathrm{R}$ Soc Trop Med Hyg 1933;27:207-10.

81. Plank HK. All parts of the desert date is toxic to snails. Annual Report USDA, AES. Puerto Rico Mayaquez, Puerto Rico; 1945. p. 24.

82. Liu HW, Nakanishi K. The structures of balanitins. Potent molluscicides isolated from Balanites aegyptiaca. Tetrahedron 1982;38:513-9.

83. Gaudin $\mathrm{O}$, Vacheriat R. Rotenone and the ichthyotoxic power in some plants of the french Sudan. Bull Sci Pharmacol 1938;45:385-94.

84. Spencer CF, Koniuszy FR, Rogers EF, Shavel JRJ, Easton NR, Kaczka EA, et al. Survey of plants for antimalarial activity. Lloydia 1947;10:145-74.

85. Ampofo O. Plants that heal. World Health Mag. WHO; 1977. p. 26-30. 
86. Schreiber FG, Schubert M. Toxicity of some chemical compounds to cercariae of Schistosoma mansoni. J Parasitol 1949;35:364-6.

87. Dhar ML, Dhar MN, Dhawan BN, Mehrotra BN, Srimal RC, Tandon JS. Screening of Indian plants for biological activity. Part IV. Indian J Exp Biol 1973;1:43-54.

88. Taniguchi M, Chapya A, Kubo, Nakanishi K. Screening of East African plants for antimicrobial activity. I. Chem Pharm Bull 1978;26:2910-3.

89. Heal RE, Rogers EF, Wallace RT, Starnes OA. Survey of plants for insecticidal activity. Lloydia 1950;13:89-162.

90. Sparg SG, Light ME, Van Staden J. Biological activities and distribution of plant saponins. J Ethnopharmacol 2004;94:219-43.

91. Dragendorff G. Die Heilpflanzen der verschiedenen Vtlker und Zeiten. F. Enke, Stuttgart; 1898.

92. Creach P. Balanites aegyptiaca and its various uses in Tchad territory. Rev Bot Appl Agric Trop 1943;20:578-93.

93. Hussein Ayoub SM, Baerheim Svendsen A. Medicinal, and aromatic plants in the Sudan. Usage and exploration. Fitoterapia 1981;52:243-6.

94. Hammiche V, Maiza K. Traditional medicine in central sahara: Pharmacopoeia of tassili N'ajjer. J Ethnopharmacol 2006; 105:358-67.

95. Iwu MM. Handbook of African Medicinal Plants. CRC Press: Boca Raton, Florida, USA; 1993.

96. Patel M. Effect of Dodonaea angustifolia on adherence of Candida albicans. Scientific meeting of the South African division of IADR. Microbiology/immunology/Infection Control, University of Witwatersrand, Wits, 2050, South Africa; 2006.

97. Mativandlela SP, Meyer JJ, Hussein AA, Houghton PJ, Hamilton CJ, Lall N. Activity against Mycobacterium smegmatis and Mycobacterium tuberculosis by an extract of South African medicinal plants. Phytother Res 2008;22:841-5.

98. Daman G, Gideon M, Patel M. Antifungal activity of a medicinal plant Dodonaea angustifolia. Scientific meeting of the South African division of IADR. Microbiology/immunology/Infection Control, University of Witwatersrand, Wits, 2050, South Africa; 2006.
99. Giday M, Teklehaymanot T, Animut A, Mekonnen Y. Medicinal plants of the Shinasha, Agew-awi and Amhara peoples in northwest Ethiopia. J Ethnopharmacol 2007;110:516-52.

100. Dominguez XA, Franco R, Cano CG, Chavez CN. Medicinal plants of Mexico. Part XLIV. Isolation of 3, 6, 40-trimethoxy-5, 7dihydroxyflavone from chapuliztle (Dodonaea viscosa var. angustifolia Jacq.). Rev Latino Am Quim 1980;11:150-1.

101. Watt JM, Breyer-Brandwijk MG. The medicinal and poisonous plants of Southern and Eastern Africa. 2nd ed. E and S. Livingstone, Ltd., London; 1981. p. 931-2.

102. Mativandlela SPN. Antituberculosis activity of flavonoids from Galenia africana phytochemistry and medicinal uses of selected medicinal plants. The university of Pretoria, chapter 3 thesis; 2009. p. 77-104.

103. Teklehaymanot T, Giday M, Medhin G, Mekonnen Y. Knowledge and use of medicinal plants by people around Debre Libanos monastery in Ethiopia. J Ethnopharmacol 2007;111:271-83.

104. Wondimu T, Asfaw Z, Kelbessa E. Ethnobotanical study of medicinal plants around 'Dheeraa' town, Arsi Zone, Ethiopia. J Ethnopharmacol 2007;112:152-60.

105. Ali NAA, Wurster M, Arnold N, Lindequist U, Wessjohann L. Essential oil composition from oleogum resin of Soqotraen Commiphora kua. Rec Nat Prod 2008;2:3:70-5.

106. Manguro LOA, Ugi I, Lemmen P. Further bisabolenes and dammarane triterpenes of Commiphora kua resin. Chem Pharm Bull 2003;51:479-82.

\section{How to cite this article}

- Fatouma Mohamed Abdoul Latif, Djaltou Aboubaker Osman, Abdirahman Elmi Fourreh, Alshaimaa Hassan Abdallah, Ali Merito, Souad Hassan, Zemede Asfaw, Ensermu Kelbessa. Candidate medicinal plant species of djiboutian pharmacopeia for testing pharmacological activities on common microbial diseases. Int J Pharm Pharm Sci 2016;8(10):78-84. 